\title{
Research on Pressure Tactile Sensing Technology Based on Fiber Bragg Grating Array
}

\author{
Jinxue $\mathrm{SONG}^{1}$, Qi JIANG ${ }^{1 *}$, Yuanyang $\mathrm{HUANG}^{1}$, Yibin $\mathrm{LI}^{1}$, Yuxi JIA ${ }^{2}$, \\ Xuewen $\mathrm{RONG}^{1}$, Rui SONG${ }^{1}$, and Hongbin $\mathrm{LIU}^{3}$ \\ ${ }^{1}$ School of Control Science and Engineering, Shandong University, Jinan, 250061, China \\ ${ }^{2}$ School of Materials Science and Engineering, Shandong University, Jinan, 250061, China \\ ${ }^{3}$ Department of Informatics, King's College London, London, WC2R 2LS, UK \\ *Corresponding author: Qi JIANGＥ-mail: jiangqi@sdu.edu.cn
}

\begin{abstract}
A pressure tactile sensor based on the fiber Bragg grating (FBG) array is introduced in this paper, and the numerical simulation of its elastic body was implemented by finite element software (ANSYS). On the basis of simulation, fiber Bragg grating strings were implanted in flexible silicone to realize the sensor fabrication process, and a testing system was built. A series of calibration tests were done via the high precision universal press machine. The tactile sensor array perceived external pressure, which is demodulated by the fiber grating demodulation instrument, and three-dimension pictures were programmed to display visually the position and size. At the same time, a dynamic contact experiment of the sensor was conducted for simulating robot encountering other objects in the unknown environment. The experimental results show that the sensor has good linearity, repeatability, and has the good effect of dynamic response, and its pressure sensitivity was $0.03 \mathrm{~nm} / \mathrm{N}$. In addition, the sensor also has advantages of anti-electromagnetic interference, good flexibility, simple structure, low cost and so on, which is expected to be used in the wearable artificial skin in the future.
\end{abstract}

Keywords: Array, tactile sensation, fiber Bragg grating, finite element analysis

Citation: Jinxue SONG, Qi JIANG, Yuanyang HUANG, Yibin LI, Yuxi JIA, Xuewen RONG, et al., "Research on Pressure Tactile Sensing Technology Based on Fiber Bragg Grating Array," Photonic Sensors, 2015, 5(3): 263-272.

\section{Introduction}

In the industrial automation system, robot is playing an increasingly important role in some high risk, high difficulty, and repetitive works in order to improve the productivity, but inevitably robots need to contact with a variety of objects in the complex work environment and will also encounter many unexpected obstacles [1], which requires the robots having good ability of tactile perception. In research, feelings of the robot like touch, pressure, slip, hot and cold sensation are called generalized tactile sensation, and the contact force sensing between the robot and object is called narrow sense of touch [2]. The tactile sensor array with the large and flexible area can cover the surface of the robot and can be used in quantitative detection of the external physical quantity, different from the human skin which can only sense the external environment in a qualitative manner [1]. In order to meet the high perception performance demand of physical

Received: 6 May 2015 / Revised version: 28 May 2015

(C) The Author(s) 2015. This article is published with open access at Springerlink.com

DOI: $10.1007 / \mathrm{s} 13320-015-0260-1$

Article type: Regular 
properties in complex environment, it is very important to study a tactile sensor with high precision, flexibility, and capability of providing enough measuring range and the spatial resolution.

As early as in the 1970s, Page et al. proposed to utilize the direct measured deformation when a soft elastic film contacts a rigid object to estimate the stiffness of the object. However, this kind of elastic film differs from the real smart skin to a great extent [3]. From the beginning of the 1980 s, the research on the robot tactile sensor has grown rapidly. Many researchers have developed tactile sensors of various sensitive principles such as piezoresistive, piezoelectric, capacitive, magnetic, and ultrasonic waveguide types [4]. But tactile sensors using above electrical principles are vulnerable to electromagnetic interference. In addition, some tactile sensors are also limited in application due to the complex production process, high cost, and difficult decoupling problems [5].

In recent years, scholars at home and abroad have been constantly exploring new theories, new methods, and new materials of the flexible tactile sensors in view of the present situation and the existent problems. Optical fiber offers an effective sensing method with small sensors for its small diameter, light weight, anti-electromagnetic interference, and suitability for large area array. These are some of the reasons why optical fibers are used in many sensing applications. In [6, 7], the researchers used the optical fiber as the transmission medium of light to sense the external tactile deformation by the mirror reflection of light intensity change instead of the electronic device or circuit, however it not only requires the complex and smart structure design but also it is easily affected by the light interference. This work focused on tactile sensors by using the fiber Bragg grating (FBG) as the sensing element without the light intensity and electromagnetic interference. And in this paper, we optimize FBG tactile sensor array units design through the ANSYS modeling and analysis which achieves good results.

\section{Tactile sensor array design}

\subsection{FBG sensing principles}

The fiber Bragg grating is a periodic structure inscribed in the core of an optical fiber. Its simple form can be defined as a periodic modulation of the refractive index along the fiber core. The FBG reflects a narrowband portion of the broadband incident light and transmits most of the rest. According to the mode coupling theory, the central wavelength of the reflected narrowband light namely $\lambda_{B}$ can be described by the Bragg condition [8]:

$$
\lambda_{B}=2 n_{\text {eff }} \Lambda
$$

where $n_{\text {eff }}$ is the effective refractive index of the single core mode of the fiber, and $\Lambda$ is the periodicity of the grating. In all of the external factors, the strain and temperature are the most important and most common factors, thus the Bragg wavelength shift due to strain and temperature can be expressed as

$$
\lambda_{B}=2 n_{\text {eff }} \Lambda=2 n_{\text {eff }}(\varepsilon, T) \Lambda(\varepsilon, T),
$$

and the full differential operation of above equation is

$$
\begin{gathered}
\frac{\Delta \lambda_{B}}{\lambda_{B}}=\left(\frac{1}{n_{\mathrm{eff}}} \frac{\partial n_{\mathrm{eff}}}{\partial \varepsilon}+\frac{1}{\Lambda} \frac{\partial \Lambda}{\partial \varepsilon}\right) \Delta \varepsilon+ \\
\left(\frac{1}{n_{\mathrm{eff}}} \frac{\partial n_{\mathrm{eff}}}{\partial T}+\frac{1}{\Lambda} \frac{\partial \Lambda}{\partial T}\right) \Delta T .
\end{gathered}
$$

The above formula is the basic sensing model of the FBG sensor. When the temperature field is zero $(\Delta T=0)$, only the axial strain $(\varepsilon)$ is taken into account, and the wavelength shift of the FBG can be given by [9]

$$
\begin{aligned}
\frac{\partial n_{\mathrm{eff}}}{n_{\mathrm{eff}}} & =-\frac{n_{\mathrm{eff}}^{2}}{2}\left[P_{12}-v\left(P_{11}+P_{12}\right)\right]\left|\varepsilon_{z z}\right| \\
P_{e} & =\frac{n_{\mathrm{eff}}}{2}\left[P_{12}-v\left(P_{11}+P_{12}\right)\right]
\end{aligned}
$$

where $P_{e}$ is the strain-optical coefficient of the optical fiber, $p_{11}$ and $p_{12}$ are two components of the 
strain-optic tensor, and $v$ is Poisson's ratio. Combining (4) and (5), the wavelength shift of the FBG is represented as

$$
\Delta \lambda_{B}=\left(1-P_{e}\right) \lambda_{B} \varepsilon
$$

For a typical optical fiber, $n_{\text {eff }}=1.456, v=0.17$, $P_{11}=0.121, P_{12}=0.270$, and $P_{e}=0.216$, therefore, the wavelength shift of the typical FBG can be given by

$$
\Delta \lambda_{B}=0.784 \lambda_{B} \varepsilon
$$

When the strain field is zero $(\Delta \varepsilon=0)$, only $T$ is taken into account, and the wavelength shift of the FBG can be given by [10]

$$
\begin{gathered}
\Delta \lambda_{B}=\lambda_{B}\left(\frac{1}{n_{\mathrm{eff}}} \frac{\partial n_{\mathrm{eff}}}{\partial T}+\frac{1}{\Lambda} \frac{\partial \Lambda}{\partial T}\right) \Delta T= \\
\lambda_{B}(\alpha+\beta) \Delta T=K_{T} \lambda_{B} \Delta T \\
\alpha=\frac{1}{\Lambda} \frac{\partial \Lambda}{\partial T} \quad \beta=\frac{1}{n_{\mathrm{eff}}} \frac{\partial n_{\mathrm{eff}}}{\partial T} \quad K_{T}=\alpha+\beta
\end{gathered}
$$

where $\alpha$ is the thermal expansion coefficient (CTE) of the optical fiber, i.e., the change in the grating period $\Lambda$ caused by the thermal expansion effect; $\beta$ is the thermo-optic coefficient of the optical fiber, i.e., the change in the refractive index $n_{\text {eff }}$ caused by the thermo-optic effect; $K_{T}$ is the fiber grating temperature sensing sensitivity coefficient.

From the above equations, once the material of the fiber grating is finalized, the temperature sensitivity coefficient and strain sensitivity coefficient associated with the material are constants to ensure a linear sensing. In this paper, one FBG packaged in the non-bearing region of the sensor is used as temperature compensation, whose wavelength is only affected by temperature; meanwhile, wavelengths of FBGs under the bearing region will change correspondingly when external load varies.

$$
\left\{\begin{array}{l}
\Delta \lambda_{B 1}=K_{T 1} \lambda_{B 1} \Delta T \\
\Delta \lambda_{B 2}=0.784 \lambda_{B 2} \mathcal{E}+K_{T 2} \lambda_{B 2} \Delta T
\end{array}\right.
$$

As we have already discussed, when the structure of FBG sensors is determined, the applied forces to the sensor can be calculated through above equations according to variations of the FBG wavelengths.

\subsection{Finite element analysis}

ANSYS software developed by America ANSYS is a large general-purpose finite element (FEA) software gathering structure, thermology, fluid, electromagnetism, and design optimization. Three-dimensional models of the coating are created using the element types SOLID95, and the material of the coating is flexible silicone, whose elastic modulus $E$ is $9.2 \mathrm{MPa}$, Poisson's ratio $v$ is 0.49 . The model of the optical fiber can be removed because its diameter is too small, and the changes in the optical fiber are equivalent to those of the coating [9]. The finite element model is shown in Fig. 1. Then the applied boundary conditions in the solution module are showed as follows. The freedom degree of the model underside is arrested in the $Y$ direction allowing it to move only in the $Z$ - and $X$-directions. In other words for the model underside, $U_{Y}=0$. Applying a vertical pressure on the upper surface of the elastic model, static analysis of the model is got. The overall deformation of the elastomer and strain of specific location can be seen in the post processing module after solution.

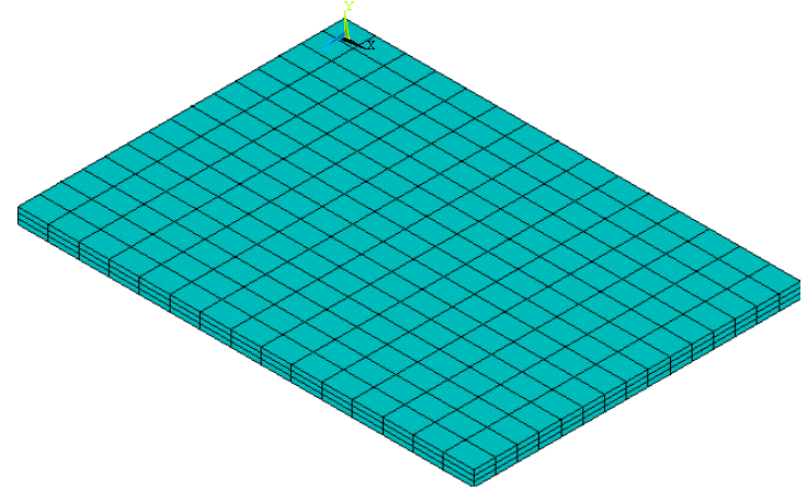

Fig. 1 Finite element model of the sensor.

Analysis of the elastomer deformation when the one-dimensional vertical pressure is applied: $F_{y}$ is increased by a step of $10 \mathrm{~N}$ from $10 \mathrm{~N}$ to $80 \mathrm{~N}$ applied to the upper surface of the elastomer, and the displacement offset of the elastomer in the $X$ direction is recorded. The central wavelength 
excursion of the FBG can be inferred from $U_{x}$ when the elastomer is stretched. As shown in Fig. 2, when the elastomer is subjected to the $Y$-direction vertical pressure, the pressure sensitivity of the sensor is $0.025 \mathrm{~nm} / \mathrm{N}$.

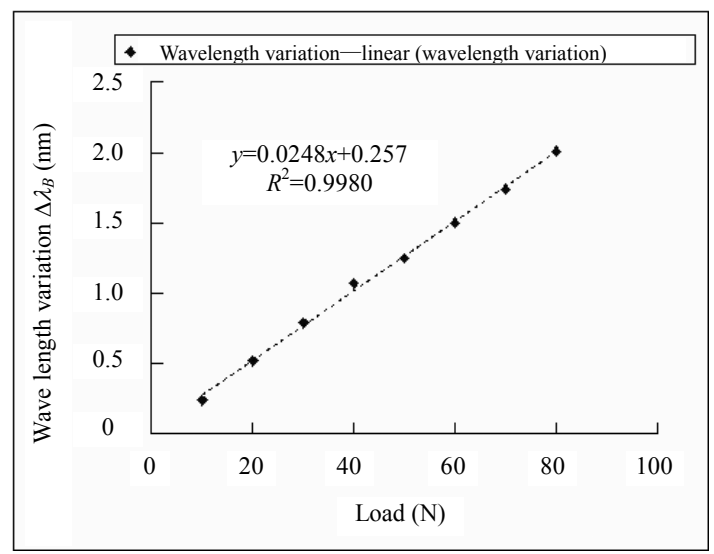

Fig. 2 Central wavelength variation under different loads.

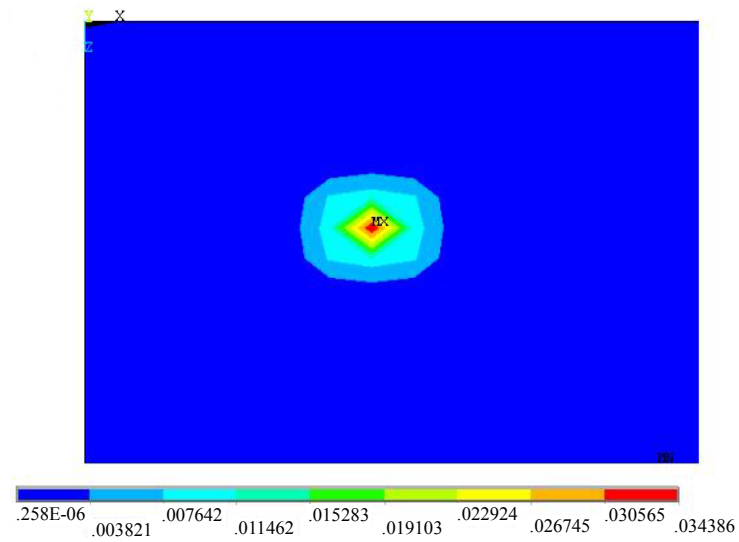

(a) Strain diagram of $2 \mathrm{~N}$

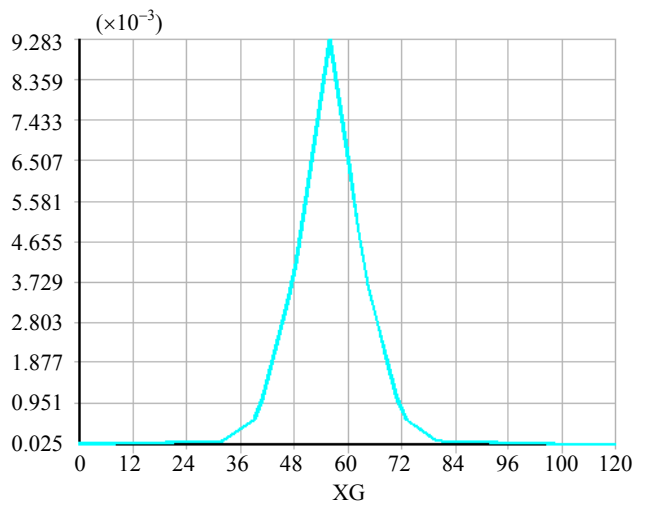

(c) $X$ direction path

Fig. 3 Path diagram of three directions.
In order to observe all parameters, $F_{y}$ is applied to the upper surface of the elastomer in an area of $1 \mathrm{~cm}^{2}$, the strain diagram is shown in Fig. 3(a). To define a path along the $Y$ direction [Fig. 3(b)], it shows that the strain is the largest, and the depth of the fiber placement is the best when the distance away from the upper surface is $1.4 \mathrm{~mm}$. We define a path along the $X$ direction to observe the change in the total displacement using the best buried depth of the optical fiber analog value [Fig. 3(c)]: the conclusion is that the optimal interval among adjacent gratings (assuming the gate length of FBG is $10 \mathrm{~mm}$ ) is $15 \mathrm{~mm}$ in the fiber axial direction. Lastly, we use the definition of the path along the $Z$ direction to observe the overall changes in the displacement [Fig.3(d)], and the best adjacent fiber spacing is $23 \mathrm{~mm}$.

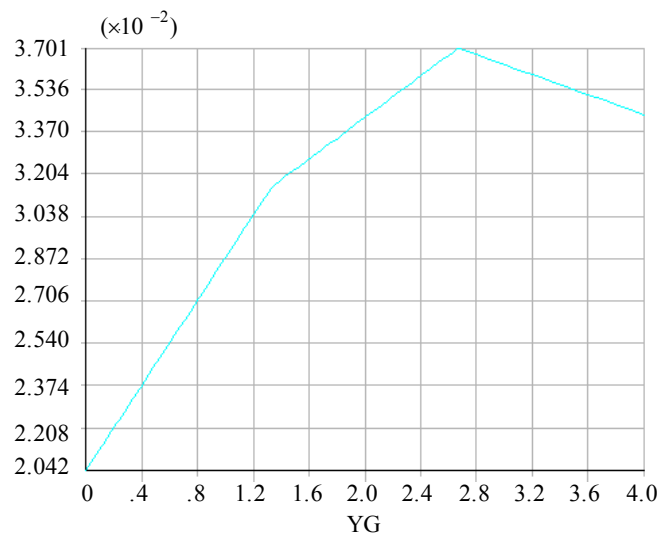

(b) $Y$ direction path

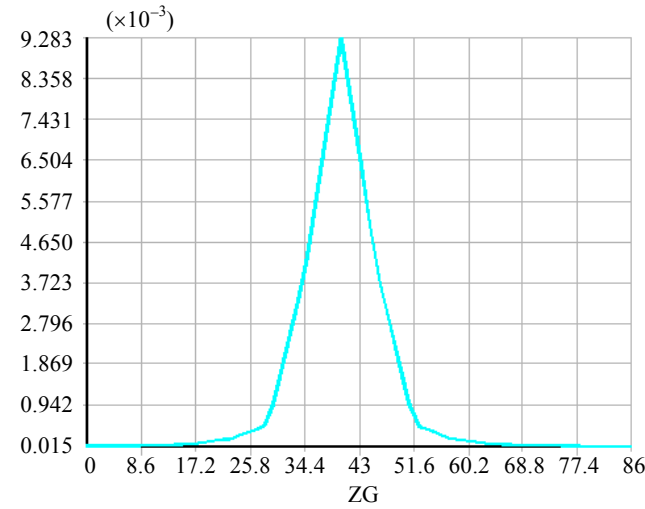

(d) $Z$ direction path 


\subsection{Structural design of the sensor}

The optimal design of the sensor structure is achieved by ANSYS simulation, and then a physical sensor is produced by the mold company. Mold is divided into two sections, each is engraved with a semicircular groove, and the diameter of the groove is $150 \mu \mathrm{m}$ (slightly larger than the diameter of the fiber). The vertical depth and lateral space of grooves are selected as the best value on the basis of ANSYS simulation. Assemble optical fibers and two parts of the mold successively, fasten mold by using matching screw, and pour the liquid

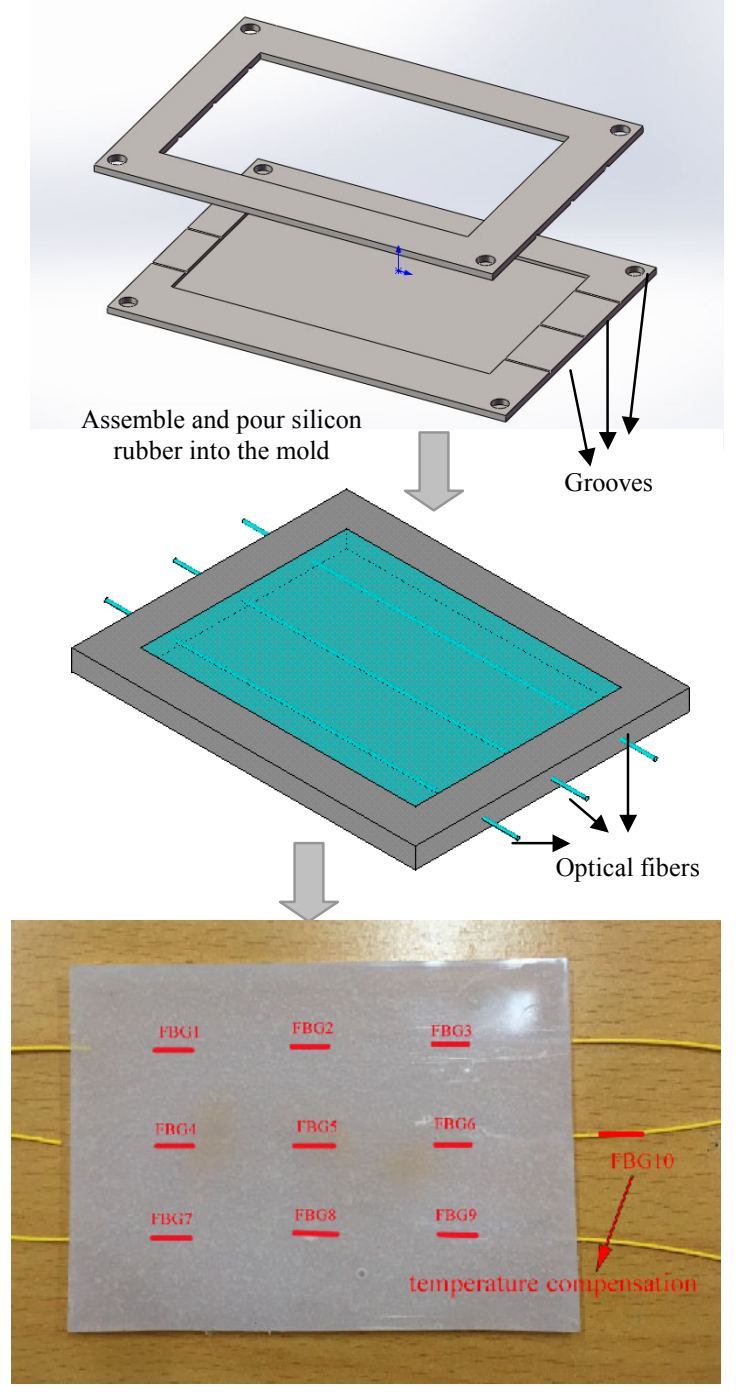

Fig. 4 Schematic of the fabrication procedure. polydimethylsiloxane and ancillary curing agent with a mass ratio $10: 1$ mixture evenly into the mold. Place the model into vacuum drying oven so mixture bubbles float to the surface and burst, and then place it at $120{ }^{\circ} \mathrm{C}$ for about an hour. As shown in Fig. 4, a sensor is achieved after mould unloading. The central wavelengths of all FBGs are shown in Table 1. The length of the gate region of these FBGs is all $10 \mathrm{~mm}$. These FBG sensing units based on the wavelength division multiplexing (WDM) technology are ideally suitable for distributed strain monitoring, as FBG sensors are easily multiplexed and offer many advantages such as linear response and relative measurement.

Table 1 Central wavelengths of FBGs.

\begin{tabular}{c|c}
\hline Number & Central wavelength $(\mathrm{nm})$ \\
\hline FBG1 & 1541.58 \\
\hline FBG2 & 1544.78 \\
\hline FBG3 & 1549.97 \\
\hline FBG4 & 1541.56 \\
\hline FBG5 & 1544.91 \\
\hline FBG6 & 1549.94 \\
\hline FBG7 & 1541.58 \\
\hline FBG8 & 1544.85 \\
\hline FBG9 & 1549.72 \\
\hline FBG10 & 1552.12
\end{tabular}

\section{Experimental test}

In order to test the effectiveness of the FBG pressure tactile sensor array, it is necessary to do the calibration experiments. Figure 5 presents the experimental test system, including SANS high precision universal testing machine and the corresponding load software, fiber grating demodulation module and its corresponding data acquisition software, and a personal computer (PC).

This article focuses on the deformation of the FBG pressure sensor under the one-dimensional vertical force [assuming the temperature field is zero $(\Delta T=0)]$. Static experiments were done by exerting a vertical pressure on FBG5 with an application area of $1 \mathrm{~cm}^{2}$ and $4 \mathrm{~cm}^{2}$, respectively. $F_{y}$ was increased or decreased by a step of $10 \mathrm{~N}$ from $0 \mathrm{~N}$ to $80 \mathrm{~N}$ for three 
cycles in the experiment. Reflection spectra of FBGs were recorded by data acquisition software, and the central wavelengths of reflection spectra were calculated by the Origin Lab data processing software. The offset and fitting curves of central wavelengths of all FBGs (the application area was $1 \mathrm{~cm}^{2}$ ) are shown in Fig. 6. The experimental results show that the sensor is basically in accordance with simulation results and has good linearity and repeatability.

Figure 7 shows wavelength variation comparison of all FBGs when exerting vertical pressure on FBG5 with the application area of $1 \mathrm{~cm}^{2}$ and $4 \mathrm{~cm}^{2}$, respectively. When the application area was $1 \mathrm{~cm}^{2}$, the Bragg wavelengths of FBG5 changed obviously, and the rest sensitive unit was not affected by the force. But when application area was $4 \mathrm{~cm}^{2}$, in addition to the change in the wavelength of FBG5
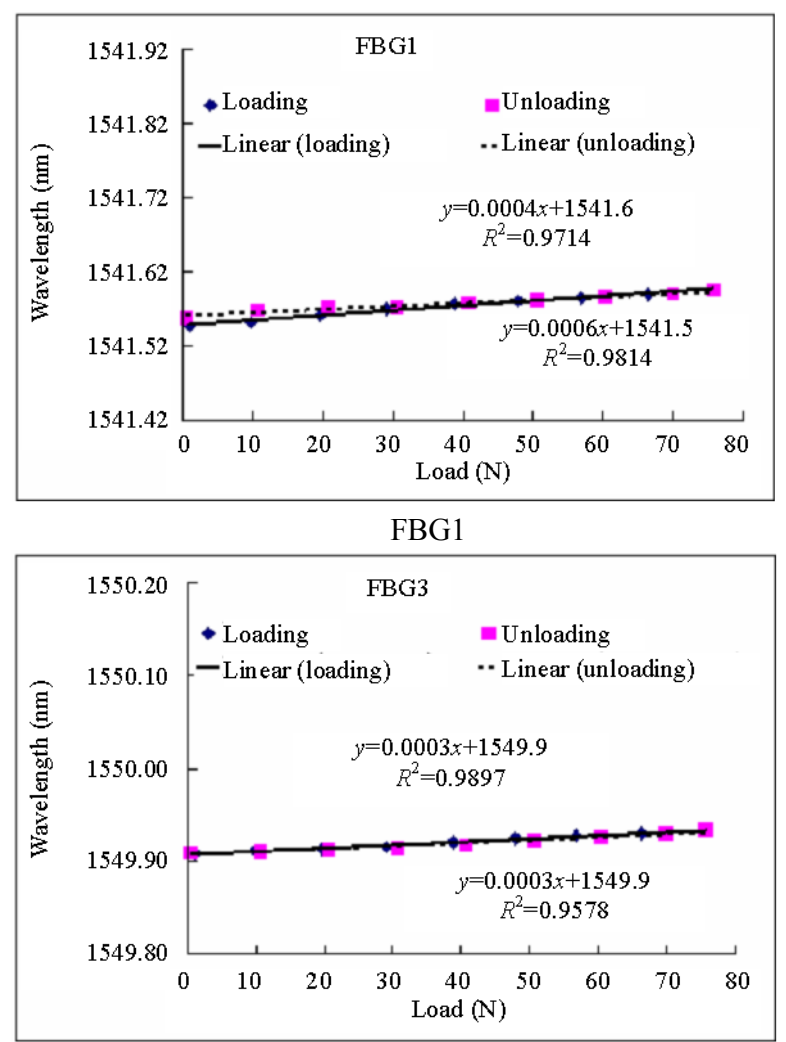

FBG3 accordingly, the central wavelength changes of the sensitive units FBG4 and FBG6 were obvious, which may be caused by the fact that FBG4, FBG5, and FBG6 by using the WDM technology were engraved on a single fiber. As shown in Fig. 8, the size and location of applied force can be visually displayed by the pseudo color map.

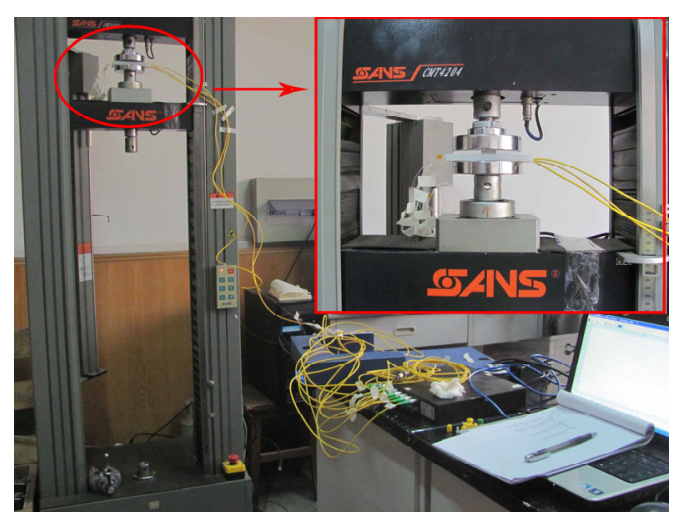

Fig. 5 Experimental site map.

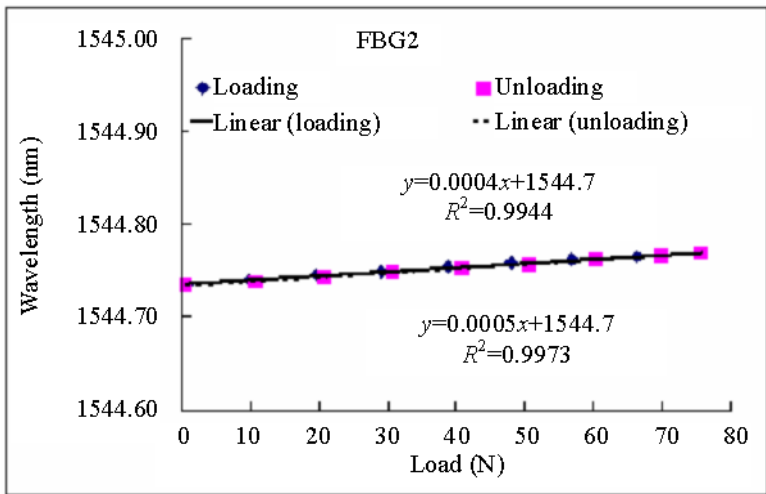

(b) FBG2

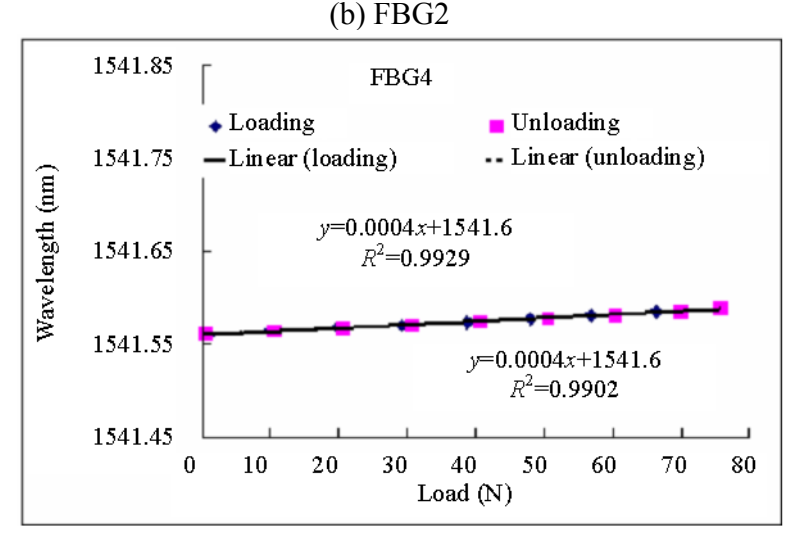

(a)

(c)

(d) FBG4 

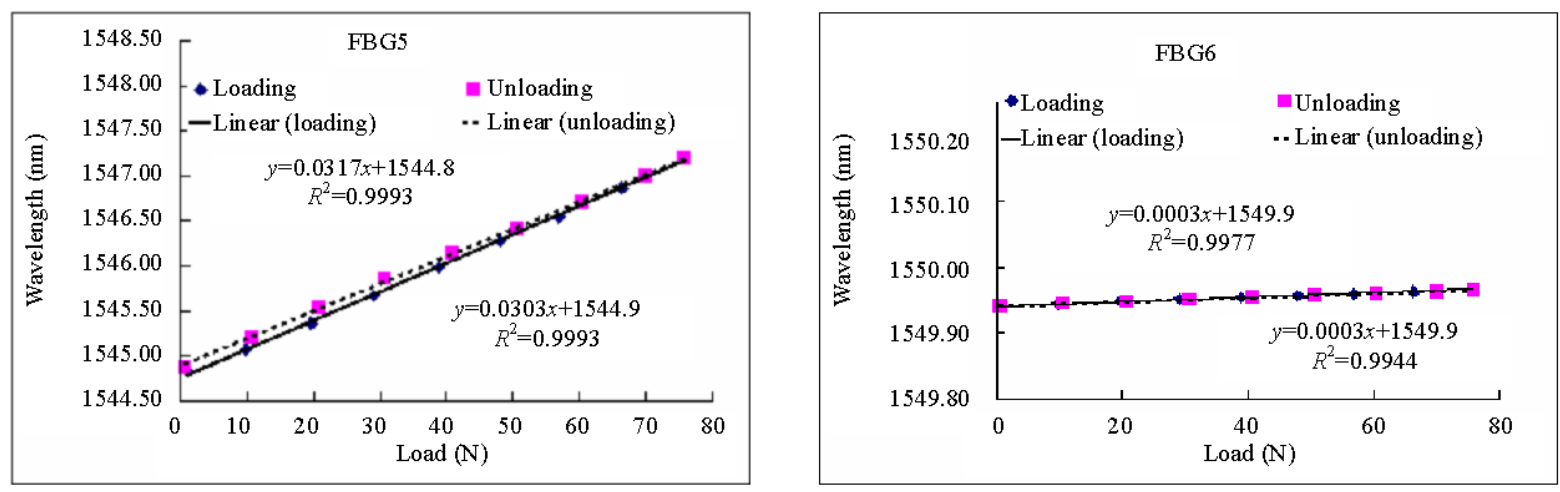

(e) FBG5

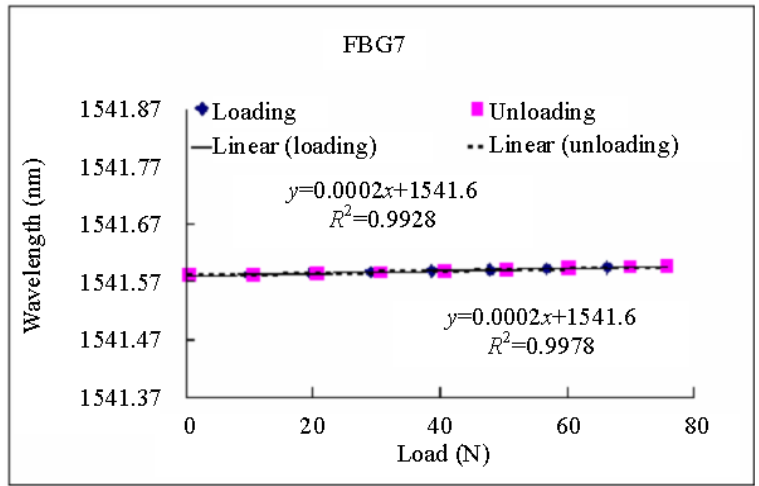

FBG7 (f) FBG6

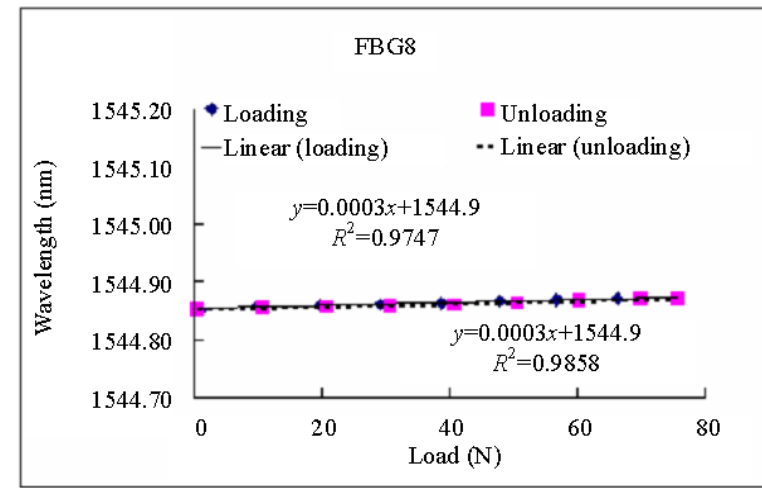

(h) FBG8 (g)

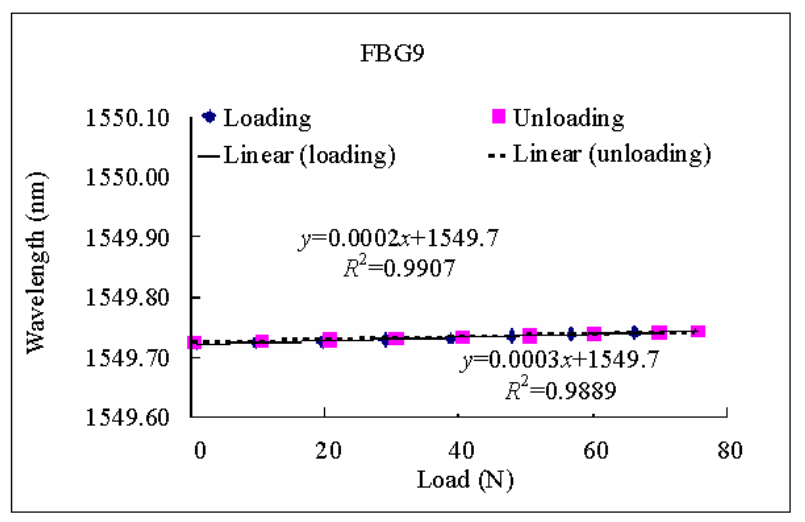

(i) FBG9

Fig. 6 Central wavelength variation of loading and unloading when applied area was $1 \mathrm{~cm}^{2}$.

When the position of applied force is not right above FBGs, the force position can be obtained after demodulating wavelength variation of all FBGs. Figure 9 shows the case when the applied force position is at a certain point in the middle of the four FBGs.

In order to simulate contact response of the tactile sensing array in application, it is needed to verify dynamic response performance of the sensor, where the square with a length of $2 \mathrm{~cm}$ was used to simulate the contact object. The dynamic testing system of the FBG pressure sensor array is shown in Fig. 10. In this experiment, the matching demodulation method was employed. When the sensor array collided with objects, the FBG sensing unit was subject to corresponding tension or compression, which would lead to the reflected spectrum offset. The electrical signal changed correspondingly after the demodulation and photoelectric conversion, and an oscilloscope was 
used to display the varied real-time signal and record the data. Use the MatLab software to display the time domain signal and frequency domain signal

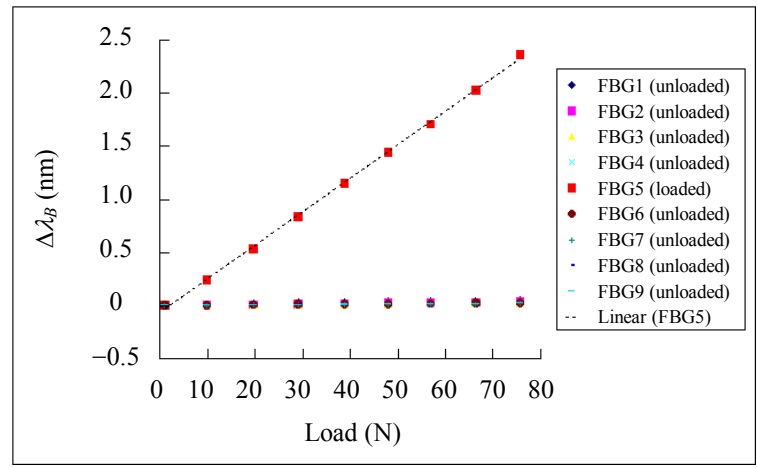

(a) $1 \mathrm{~cm}^{2}$

Fig. 7 Central wavelength variation of different applied areas.

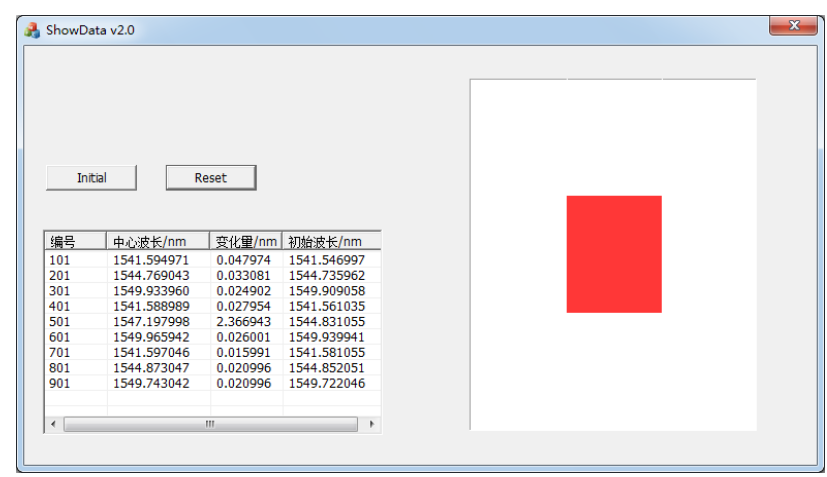

(a) $1 \mathrm{~cm}^{2}$

Fig. 8 Pseudo-color picture

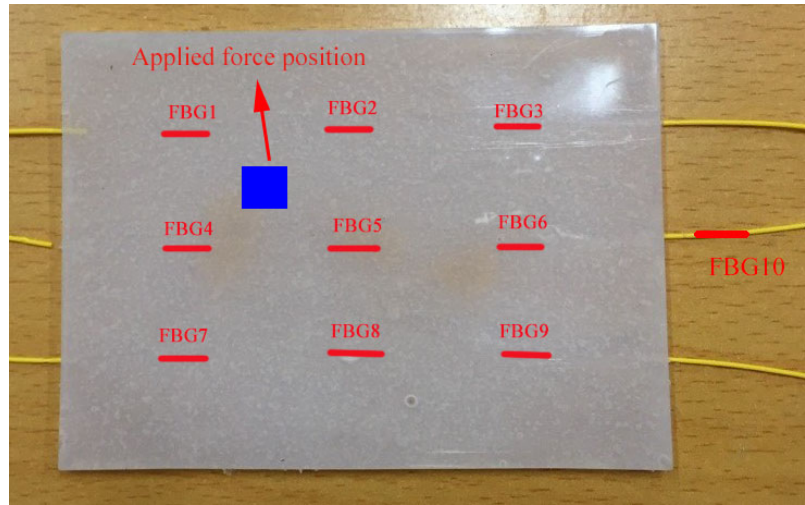

(a) Diagrammatic sketch after filtering and Fourier transform. As shown in Fig. 11, it is known that when the tactile array sensing element receives an excitation after the collision, the FBG can response timely, and the excitation frequency is close to $5 \mathrm{~Hz}$.

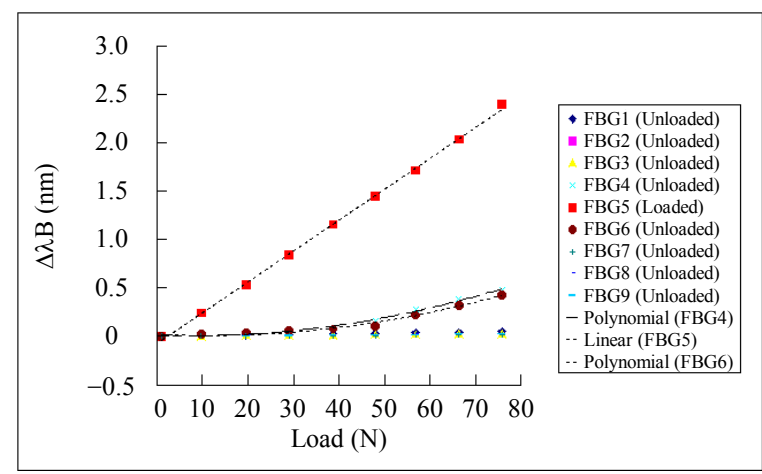

(b) $4 \mathrm{~cm}^{2}$

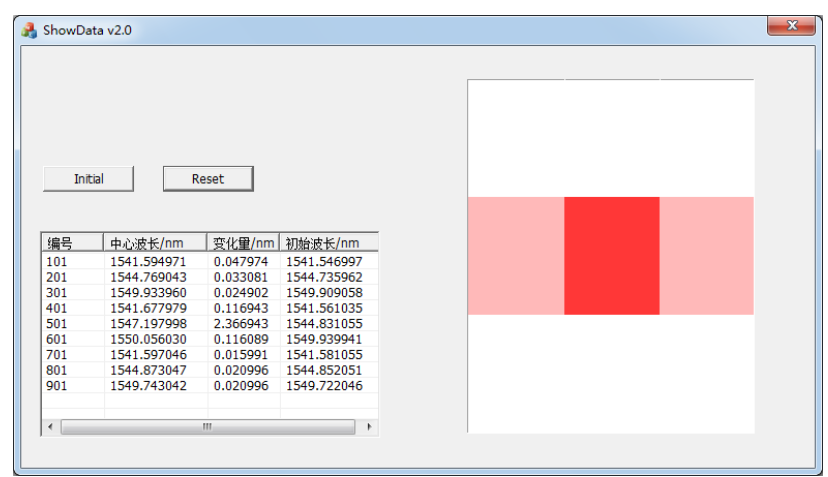

(b) $4 \mathrm{~cm}^{2}$

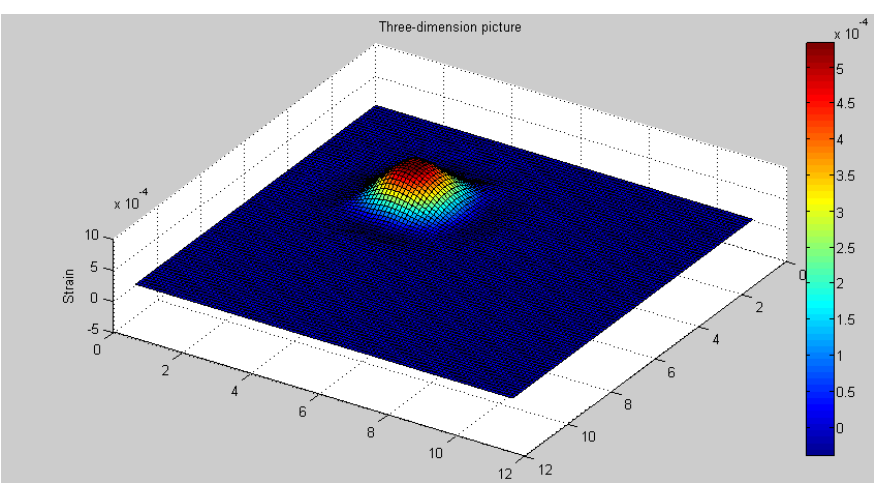

(b) Three-dimension pictures

Fig. 9 Force $\left(F_{y}=20 \mathrm{~N}\right)$ was applied on FBG1, FBG2, FBG4, and FBG5 of the FBG pressure sensor array. 


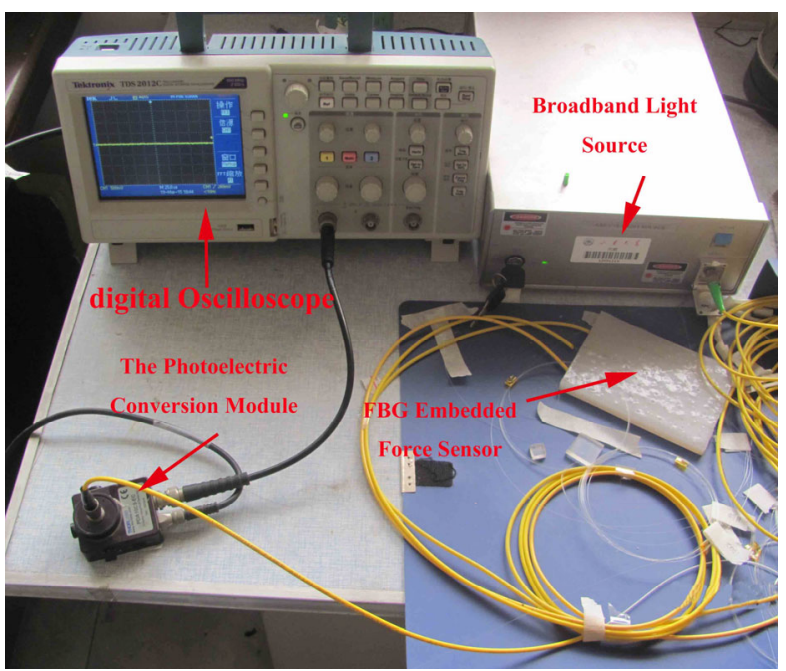

Fig. 10 Dynamic contact experiment of the sensor.
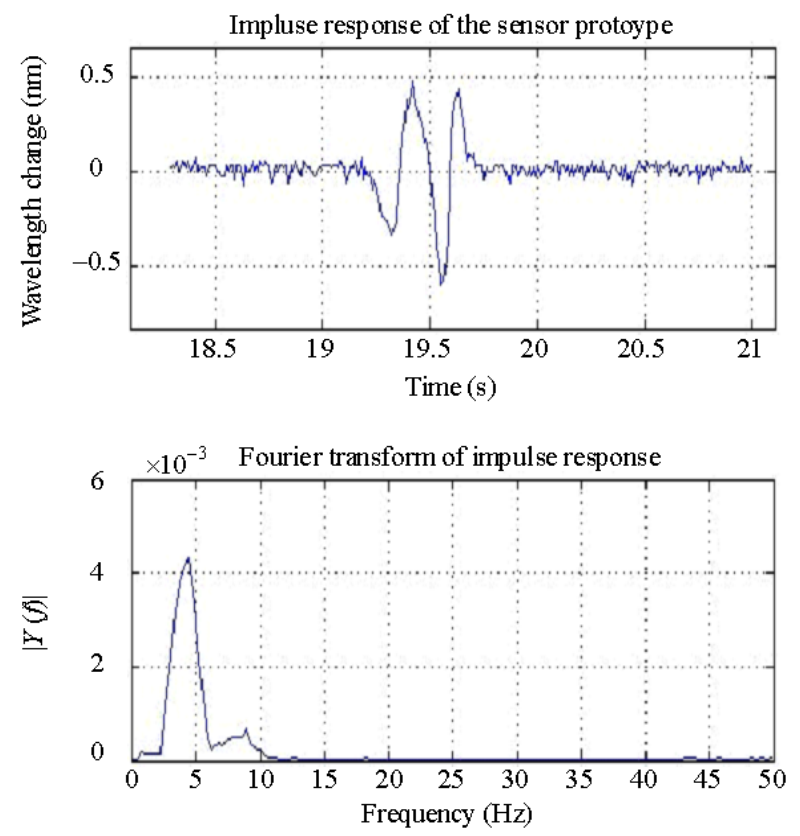

Fig. 11 Dynamic response curve.

\section{Conclusions}

According to the hot issues of robot artificial skin tactile sensing, this paper researches on the simulation, analysis, preparation, and testing technology of the FBG array pressure sensor. The new pressure tactile sensor has the advantages of high sensitivity and good linearity, and it is expected to produce a large area and array artificial skin to provide a new possible application in the robot wearable skin technology. But the results of the present study still has the issues that the spatial resolution of the sensor is not high enough, the size and the structure still need further miniaturization and so on. Therefore, we will continue to improve the work in the future.

\section{Acknowledgment}

This work was financially supported by China NSFC (No. 61271073 and No. 61473175) and was supported by the Fundamental Research Funds of Shandong University (No. 2015JC040).

Open Access This article is distributed under the terms of the Creative Commons Attribution License which permits any use, distribution, and reproduction in any medium, provided the original author(s) and source are credited.

\section{References}

[1] Y. Huang, W. Lu, X. Zhao, C. Lian, and Y. Ge, "Design and experiment of flexible multi-functional tactile sensors for robot skin," ROBOT, 2011, 33(3): 347-359.

[2] S. Liu, W. Huang, A. Wang, and A. Song, "Overview and prospect of research and development on robot tactile sensor technology," ROBOT, 2002, 24(4): 362-266.

[3] C. J. Page, A. Pugh, and W. B. Heginbotham, "New techniques for tactile imaging," Radio and Electronic Engineer, 1976, 46(11): 519-526.

[4] M. W. Strohmayr, H. Worn, and G. Hirzinger, "The DLR artificial skin step I: uniting sensitivity and collision tolerance," in 2013 IEEE International Conference on Robotics and Automation, Karlsruhe, Germany, pp. 1012-1018, 2013.

[5] V. A. Ho, M. Makikawa, and S. Hirai, "Flexible fabric sensor toward a humanoid robot's skin: fabrication, characterization, and perceptions," IEEE Sensors Journal, 2013, 13(10): 40654079.

[6] R. Ahmadi, M. Packirisamy, J. Dargahi, and R. Cecere, "Discretely loaded beam-type optical fiber tactile sensor for tissue manipulation and palpation in minimally invasive robotic surgery," IEEE Sensors Journal, 2012, 12(1): 22-32. 
[7] H. Xie, A. Jiang, L. Seneviratne, and K. Althoefer, "Pixel-based optical fiber tactile force sensor for robot manipulation," in 2012 IEEE Sensors, Taipei, pp. 1-4, 2012.

[8] S. F. O. Silva, O. Frazão, J. L. Santos, F. M. Araújo, and L. A. Ferreira, "Discrimination of temperature, strain, and transverse load by using fiber Bragg gratings in a twisted configuration," IEEE Sensors Journal, 2012, 6(6): 1609-1613.
[9] K. O. Hill, "Photosensitivity in optical fiber waveguides: from discovery to commercialization," IEEE Journal of Selected Topics in Quantum Electronics, 2000, 6(6): 1186-1189.

[10] Q. Jiang, H. Du, and D. Hu, "Hydraulic pressure sensor based on fiber Bragg grating," Optical engineering, 2011, 50(6): 064401064404. 